\title{
EIN MODELL ZUR MEHRPERIODIGEN WERTPAPIERPLANUNG
}

\author{
Klaus Hellwig, Universität Ulm
}

Im Gegensatz zu den herkömmlichen, auf einer Anwendung des Bernoulli-Prinzips basierenden Modellen der Portfolioplanung wird ein Ansatz formuliert, der ohne explizite Vorgabe einer Risikonutzenfunktion auskommt. Statt dessen wird eine Planung auf der Grundlage zweier Grundprinzipien - Arbitragefreiheit und Erhaltung des Ertragswertes - vorgeschlagen. Neben dem Verzicht auf die Ermittlung und Aggregation geeigneter Nutzenfunktionen bietet der Ansatz ein Lösungskonzept, das insbesondere für Investmentgesellschaften geeignet erscheint, die im Interesse ihrer Anleger die finanzielle Leistungsfähigkeit der von ihnen verwalteten Fonds erhalten wollen.

Zum Nachweis der Existenz einer den Grundprinzipien genügenden Lösung wird das Modell in ein Fixpunktproblem transformiert. Dabei wird unterstellt, daß das Entscheidungsproblem durch einen endlichen Zustandsbaum charakterisiert werden kann. Ferner wird angenommen, daß in jedem Zustand endlich viele beliebig teilbare Anlagealternativen bestehen, wobei Leerverkäufe zugelassen sind. Mit Hilfe des Fixpunktsatzes von Kakutani wird eine Lösung des Problems unter sehr allgemeinen Voraussetzungen nachgewiesen. Für die Lösung werden einige Eigenschaften abgeleitet und diskutiert. Insbesondere kann gezeigt werden, daß das Modell für den Fall einer einperiodigen Planung mit dem Modell der renditeorientierten Portfolioplanung übereinstimmt (vgl. Hellwig, K., Renditeorientierte Portfolioplanung, Zeitschrift für Betriebswirtschaft, Januar 1993). Für diesen Fall können damit die dort gewonnenen Ergebnisse, wie die Kompatibilität mit Bernoulli - Prinzip bei logarithmischer Nutzenfunktion, übertragen werden.

Für den Fall, daß nicht nur die Erhaltung, sondern das Wachstum des Ertragswertes mit a priori gegebenen (u. U. zustandsabhängigen) Wachstumsraten gewünscht wird, lassen sich entsprechende Ergebnisse ableiten. 